\title{
Beneficios de los ácidos grasos poliinsaturados de cadena larga n-3 en la enfermedad por hígado graso no alcohólico
}

\author{
Benefits of $\mathrm{n}-3$ long-chain \\ polyunsaturated fatty acids in \\ non-alcoholic fatty liver disease
}

\begin{abstract}
Nonalcoholic fatty liver disease (NAFLD) is commonly associated with the clinical features of the metabolic syndrome including obesity, insulin resistance and dyslipidemia. NAFLD. Is of clinical relevance because its high prevalence $30 \%$ of the general population) and broad spectrum of histological damage, ranging from simple steatosis that is generally non progressive, to nonalcoholic steatohepatitis which can lead to cirrhosis, hepatoceIlular carcinoma, and liver failure. At present, different factors have been identified that lead to this liver disease, highlighting the high content of free fatty acids and insulin resistance. In this regard, excess of free fatty acids caused by a high intake of saturated fatty acids, trans fatty acids and of carbohydrates as well the increased formation free radicals that stress the endoplasmic reticulum, can trigger liver lipotoxicity. Regarding fatty acids, $n-3$ long-chain polyunsaturated fatty acids ( $n-3$ LCPUFA) have been associated to many benefits for human health. n-3 LCPUFA, such as EPA and DHA, have protective roles in cardiovascular health and in the functionality and integrity of the central nervous system. Currently, the possible therapeutic uses of these fatty acids is expanding, attributing their positive effects not only for the treatment of cardiovascular and neurodegenerative diseases, but also seeing it as an effective alternative in the management of NAFLD. The present review analyzes the potential use of $n-3$ LCPUFA in the treatment and protection of NAFLD.

Key words: Nonalcoholic liver disease, dyslipidemia, lipotoxicity, n-3 long-chain polyunsaturated fatty acids.
\end{abstract}

María Catalina Hernandez-Rodas (1) Jessica Morales P. (1) Rodrigo Valenzuela B. $(1,2)$ Gladys Morales I. (3) Alfonso Valenzuela B. $(2,4)$

(1) Departamento de Nutrición Facultad deMedicina Universidad de Chile Santiago, Chile (2) Laboratorio de Lípidos, INTA, Universidad de Chile, Santiago, Chile. (3) Departamento de Salud Pública, Facultad de Medicina, Universidad de la Frontera, Temuco, Chile. (4) Facultad de Medicina, Universidad de los Andes, Santiago, Chile.

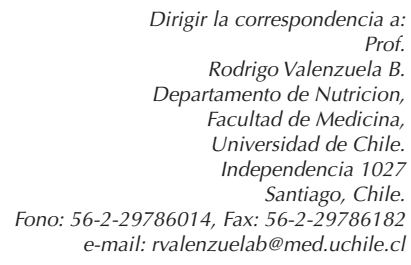

ste trabajo fue recibido el 5 de Octubrede 2015 , aceptado con modificaciones el 14 de Enero de 2016 y aceptado para ser publicado el 20 de Abril de 2016.

\section{INTRODUCCIÓN}

Los ácidos grasos poliinsaturados de cadena larga $n-3$ (AGPICL $n-3$ ) son nutrientes esenciales para el crecimiento y el desarrollo de los mamíferos, incluyendo los seres humanos, efecto demostrado por numerosas investigaciones iniciadas con el trabajo pionero de los norteamericanos George y Mildred Burr en los inicios del siglo XX (1). Burr y Burr (1929) observaron que una alimentación carente en grasas originaba en la rata alteraciones cutáneas y dermatitis principalmente en la cola, ocasionando posteriormente la muerte. Aunque estos investigadores no pudieron determinar cuáles eran los componentes dietarios cuya ausencia producía estos efectos, pudieron observar que la adición de una pequeña cantidad de grasa láctea o de un homogenizado hepático a la dieta revertía estos síntomas. Más tarde, con la cromatografía gaseosa, fue posible separar e identificar los diferentes tipos de ácidos grasos, descubriéndose que el ácido linoleico (C18:2 n-6, AL) y el ácido $\alpha$-linolénico (C18:3 n-3, ALA) eran los componentes deficitarios en la dieta utilizada por los Burr. Hansen y cols. el año 1958 (2), demostraron la esencialidad de estos ácidos grasos y la de sus derivados metabólicos y postulando sus efectos benéficos en la salud.

En los últimos años se ha incrementado la importancia fisiológica de los AGPICL n-3, especialmente del ácido eicosa- 
pentaenoico (C20:5 n-3, EPA) en la prevención de las enfermedades cardiovasculares y la del ácido docosahexaenoico (C22:6 $\mathrm{n}-3, \mathrm{DHA}$ ) en el desarrollo y función del sistema nervioso (3). El EPA y el DHA se han asociado con otros efectos positivos en la salud (4-5), en la artritis reumatoide (6), obesidad (7), diabetes mellitus (8), enfermedades neurodegenerativas (9), asma (10), enfermedad inflamatoria intestinal (11), algunos cánceres (12-13), falla renal crónica (14) y daño cardíaco y hepático causados por episodios de isquemia/reperfusión (15).

El hígado es un órgano particularmente afectado por una dieta con altos niveles de ácidos grasos saturados (AGS), ácidos grasos trans (AGT), y por otros componentes dietarios que pueden saturar su función desintoxicante, lo que conduce a trastornos metabólicos y daño hepático, tales como la enfermedad por hígado graso no alcohólico (EHGNA), la que puede progresar desde esteatosis (enfermedad benigna y no progresiva) hasta esteatohepatitis no alcohólica (EHNA) que puede evolucionar a fibrosis y cirrosis (resultado final del daño crónico al hígado). La esteatosis hepática corresponde a la acumulación intracelular de lípidos y la subsecuente formación de gotas lipídicas en el citoplasma de los hepatocitos, asociada a un incremento del tamaño del hígado (hepatomegalia). Cuando la esteatosis se acompaña por inflamación, la condición se denomina esteatohepatitis no alcohólica (EHNA). Ambas patologías se agrupan bajo el término de enfermedad por hígado graso no alcohólico (EHGNA) (16). Así, la EHGNA se define como un almacenamiento de triglicéridos (TG) en los hepatocitos sobre $5 \%$ del peso del órgano, en ausencia de un consumo mayor a $20 \mathrm{~g}$ de alcohol/día. Se vincula con diversas características del síndrome metabólico (SM), destacándose principalmente la resistencia a insulina (RI) (17) y alteraciones en los niveles hepáticos de ácidos grasos libres (AGL) (18). Considerando que la EHGNA es una enfermedad crónica cada vez más común, vinculada con patologías de gran impacto en salud como lo es la diabetes y obesidad, la búsqueda de nuevos agentes nutricionales para la prevención y el tratamiento de esta enfermedad se consideran una prioridad en salud pública. En este sentido, los AGPICL n-3 son un foco de interés en la EHGNA dado los múltiples efectos cito-protectores que les atribuye. Esta revisión analiza los mecanismos metabólicos involucrados en el desarrollo de la EHGNA, como lo son la lipotoxicidad inducida por los AGS y por los AGT, el aumento de las especies reactivas del oxígeno (EROs) y el estrés del retículo endoplasmático (RE), y discute el posible papel protector de los AGPICL n -3 como una alternativa en el tratamiento de esta enfermedad.

\section{EHGNA Y MECANISMOS IMPLICADOS EN SU DESARROLLO}

La EHGNA es una enfermedad cada vez más común en el mundo desarrollado, proyectándose a ser la principal causa de trasplante hepático (19). La importancia clínica de esta enfermedad se debe a su elevada prevalencia (30\% de la población general) (20) y a su amplio espectro de daño histológico, que va desde esteatosis simple, generalmente no progresiva en los hepatocitos (acumulación hepática de triglicéridos $>5,5 \%$ del peso del tejido, utilizando imágenes de resonancia magnética (21-22), o > $5 \%$ correspondiente a 50 $\mathrm{mg} / \mathrm{g}$ en peso húmedo), a una lesión hepática más importante que conduce a inflamación lobular, fibrosis, cirrosis, carcinoma hepatocelular y esteatohepatitis no alcohólica (23). La EHGNA se origina cuando no hay una coordinación entre la secreción de las lipoproteínas de muy baja densidad (VLDL) y la síntesis de lípidos, lo que produce un desequilibrio en el depósito de
TG y el aumento tisular de AGL. La predisposición genética y ciertos factores como la sobrealimentación, el alto consumo de fructosa (24), la obesidad (25), la inhibición de la actividad de la proteína microsomal de transferencia de triglicéridos (MTP) (26-27), el estrés oxidativo hepático (28), la peroxidación de lípidos (29) y la RI (30), conllevan a un exceso de AGL, desencadenando esta enfermedad. Existe importante evidencia que apunta al alto contenido de AGL y la RI como los principales factores de inicio o de "primer golpe" de la EHGNA (31-32). Además, se plantea que la inflamación desempeña un papel importante en el denominado "segundo golpe", con aumento de la producción de EROs y de citoquinas pro-inflamatorias (33). La información epidemiológica, además, demuestra que la EHGNA se asocia con dislipidemia, (bajas concentraciones de colesterol-HDL, aumento de TAG séricos y de las partículas de colesterol-LDL con morfología aterogénica) (34-37), de manera que esta enfermedad es considerada por muchos autores como la manifestación hepática del SM (38-39). De igual modo, la gravedad de la enfermedad se correlaciona positivamente con la acumulación de tejido adiposo visceral, tanto en sujetos obesos y no obesos, sugiriendo que la infiltración de grasa hepática puede estar influenciada por adipoquinas, producidas por la grasa visceral, las cuales tienen un papel esencial en la modulación de la señalización de insulina y en la inflamación en forma independiente del índice de masa corporal (40). El crecimiento del tejido adiposo es especialmente importante ya que libera AGL a la circulación portal, es decir, directamente al hígado, desencadenando diversas alteraciones metabólicas que facilitan el desarrollo de la EHGNA. La figura 1 esquematiza los cambios metabólicos inducidos por la EHGNA.

Los mecanismos metabólicos relacionados con el desarroIlo de EHGNA, se inician por el desequilibrio entre el aporte y la degradación de los lípidos. Los lípidos almacenados en el tejido adiposo que fluyen al hígado como AGL, los que son sintetizados por el propio hígado mediante la lipogénesis de novo y los ácidos grasos procedentes de la dieta, son las principales fuentes lipídicas que en condiciones fisiológicas contribuyen a formar la grasa hepática. Como consecuencia de la ingesta alimentaria se utiliza los carbohidratos preferentemente para la formación de ATP, pero cuando la ingesta de carbohidratos es excesiva, la vía de la lipogénesis de novo permite su conversión en ácidos grasos los que a su vez son esterificados a TAG en el hígado, y posteriormente almacenados como gotas lipídicas en los hepatocitos, secretados a la sangre como VLDL o hidrolizados nuevamente a AGL para su oxidación. La infiltración de grasa en el hígado puede producirse por alguna de las siguientes causas: i) aumento en la cantidad de AGL que alcanzan el hígado por la vía portal, ii) incremento en la lipogénesis de novo y iii) disminución de la $\beta$-oxidación de los ácidos grasos y/o de la secreción de TAG en forma de VLDL. Donnelly y cols. (2005) (41) demostraron en pacientes con EHGNA que 60\% de los TAG hepáticos provienen de los AGL circulantes, 25\% deriva de la lipogénesis de novo y $15 \%$ restante se forma a partir de los AGL procedentes del metabolismo de la grasa dietaria. El elevado aporte de AGL al hígado y el incremento en la síntesis, serían las principales causas del almacenamiento de grasa en la esteatosis, mientras que la alteración de las vías de oxidación de los ácidos grasos y/o de la secreción de VLDL tendría una menor incidencia.

\section{EHGNA, DISLIPIDEMIA Y SINDROME METABÓLICO}

La presencia de dislipidemia (hipercolesterolemia, hipertrigliceridemia, o ambas) se reporta en 20 a $80 \%$ de los casos asociados con hígado graso no alcohólico (42). El contenido 
de grasa en el hígado, refleja el equilibrio entre el flujo de AGL a través de la lipólisis, la lipogénesis, la oxidación de ácidos grasos y la secreción de VLDL. El depósito anormal de TAG hepáticos es probablemente una consecuencia de la saturación de la oxidación de ácidos grasos y de la secreción de VLDL. Sin embargo, el mayor porcentaje proviene de los $A G L$, lo que sugiere que la dislipidemia es uno de los factores de riesgo más frecuentes para el desarrollo y severidad de la EHGNA (43). Esta alteración metabólica ocurre posterior al consumo de alimentos principalmente compuestos por grasas, lo que genera un aumento de los lípidos postprandiales en sangre (hiperquilomicronemia). Este mecanismo se encuentra alterado en las personas que presentan dislipidemia, ya que se produce una hiperlipidemia postprandial, la que junto con los AGL causa efectos colaterales que empeoran la enfermedad hepática (44). Los quilomicrones al alcanzar la circulación sistémica, a través de los vasos linfáticos en lugar de la vena portal, entregan al tejido adiposo una alta cantidad de AGL para su almacenamiento como TAG; lo que contribuye directamente a la infiltración de TAG en la pared arterial y en la formación de una placa ateromatosa con aumento de la permeabilidad endotelial, o indirectamente después de la liberación de productos de lipólisis tales como $A G L$, causando la activación de vías de señalización pro-inflamatorias en las células endoteliales (45).

Un $85 \%$ de los sujetos que presentan EHGNA en comparación con $30 \%$ de los casos control, presentan RI y tienen un metabolismo anormal de la glucosa, es decir, prediabetes o diabetes tipo 2 (46). La RI es un factor primordial en el desarrollo y progresión de la enfermedad, sugerida como el primer acontecimiento causante de un aumento del flujo de $A G L$ al hígado, derivado de la reducción del efecto supresor de la lipólisis ejercido por la insulina en el tejido adiposo (47). En la EHGNA, el hígado se hace resistente a la acción de la hormona, incrementando así la gluconeogénesis y la glucogenolísis (47). El aumento en la producción de glucosa hepática, acoplada a su menor captación periférica, acentúa la hiperglicemia y la secreción de insulina estimulando aún más la lipogénesis hepática (48). La hiperglicemia promueve, además, la liberación de factores inflamatorios tales como el fibrinógeno y la proteína $C$ reactiva (PCR) (49-51). No está claro si la RI hepática es una causa o consecuencia de la esteatosis (52). Se ha confirmado que la hipertrigliceridemia está presente en $64 \%$ de las personas con esta enfermedad (53), donde la RI en el tejido hepático desempeña un papel crucial, ya que provoca el incremento de la síntesis de TAG y una disminución de la oxidación de ácidos grasos, situación observada en pacientes con SM en los que se produce un incremento de las VLDL y una reducción del colesterol-HDL. La prevalencia del SM en la EHGNA se ha estimado que varía desde el $18 \%$ en sujetos con peso normal al $67 \%$ en sujetos obesos (54-55).

La hormona adiponectina, secretada por el tejido adiposo, se encuentra directamente relacionada con el SM, y sus bajas concentraciones se asocian con EHGNA, en comparación con sujetos controles (56). Esta hormona es de gran relevancia, ya que participa en el metabolismo de la glucosa y de los ácidos grasos. Se ha demostrado que aumenta la sensibilidad a la insulina en el hígado, en el músculo esquelético y en el tejido adiposo; además, activa la proteína quinasa activada por AMP cíclico (AMPK) y el receptor activado por proliferadores peroxisomales - alfa (PPAR- $\alpha$ ), estimulando la oxidación de ácidos grasos en el hígado y en el músculo (57). La activación de PPAR- $\alpha$ induce la expresión de genes involucrados en la ß-oxidación (58-59) y por ello, su inactivación puede inducir

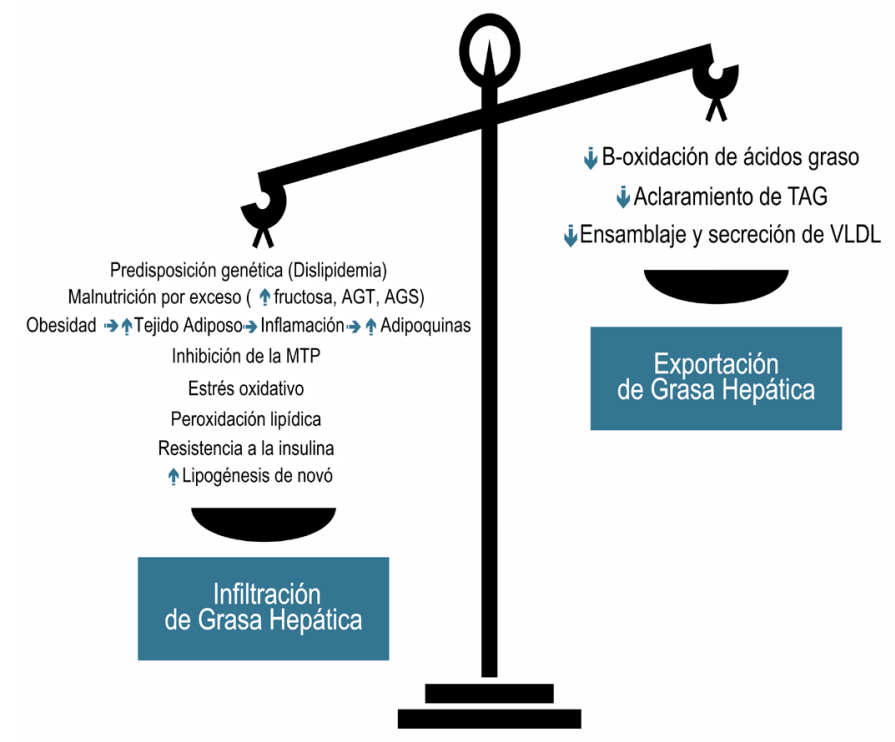


EHGNA, observada en ratones knock-out en condiciones de ayuno o alimentados con una dieta alta en grasa (DAG) (60-61). Los niveles de adiponectina se correlacionan inversamente con los TAG en el plasma y positivamente con el tamaño y los niveles de colesterol-HDL y LDL, lo que apunta a su papel protector contra el SM, específicamente contra la RI y la dislipidemia (62). Cuando la sobrecarga lipídica aumenta y los mecanismos compensatorios son sobrepasados, se produce apoptosis de los hepatocitos e inflamación. La acumulación anormal de AGL hepática puede conllevar a mecanismos de hepatotoxicidad, como la lipotoxicidad y lipoapotosis hepática, que a su vez pueden ser estimulados por el depósito de AGS, AGT, el aumento de EROs y el estrés del RE (63).

\section{LIPOTOXICIDAD DE ÁCIDOS GRASOS SATURADOS Y TRANS}

La lipotoxicidad hepática es un fenómeno que se caracteriza por el aumento de los AGL y su almacenamiento como grasa ectópica. Esto origina la activación de diversas vías metabólicas que provocan desacoplamiento celular con la generación de cascadas de señalización relacionadas con el inicio de la apoptosis de las células afectadas y finalmente terminan con la falla funcional del hígado. El aumento de la ingesta de AGT, es constantemente un foco de interés científico. La relación entre la obesidad originada por el alto consumo de AGS y AGT, no está esclarecida. La alta ingesta dietética de estos ácidos grasos aumenta el peso del hígado y el contenido de TAG hepáticos, los cuales se almacenan en el tejido adiposo causando alteraciones en los niveles de colesterol plasmáticos, observado en el modelo animal (64). Los AGT son estructuralmente similares a los AGS, ubicándose principalmente en el carbono 1 (sn-1) de los TAG y por lo tanto ejercen su influencia de la misma manera que los AGS sobre la estructura y función de las membranas, disminuyendo así su fluidez (65). En animales se ha demostrado que los AGT dietarios son detectados en el tejido adiposo y en los fosfolípidos de las distintas membranas celulares, ocupando las posiciones normalmente utilizadas por los AGS, además modifican negativamente el perfil lipídico, aumentando los niveles de colesterol-LDL y TAG y reduciendo los niveles del colesterol-HDL (65). El exceso de ambos tipos de ácidos grasos es nocivo para la salud cardiovascular (65). Los AGT han demostrado, en modelos animales, ser un factor importante en el desarrollo de la EHGNA (66). El aumento de la peroxidación de lípidos hepáticos, indicador de estrés oxidativo, se ha demostrado en el hígado de ratas alimentadas con alta ingesta de AGT, lo que sugiere que podría ser la base del desarrollo de EGHNA (67). Se ha demostrado en ratones que la ingestión a largo plazo de AGT aumenta los TAG hepáticos debido al aumento de la actividad y la expresión de las enzimas lipogénicas y eleva la expresión de la proteína de unión al elemento de respuesta a esteroles -1c (SREBP-1c) (68). Esta proteína, en respuesta al aumento de la insulina y de la glucosa, induce la expresión de los genes que codifican para las enzimas lipogénicas y glucolíticas, lo que aumenta los depósitos de grasa, que junto a los AGL circulantes captados por el hígado, dan lugar a la esteatosis.

Se ha demostrado la hepatotoxicidad generada por los AGS (69-70). La interrupción en la síntesis de TG, debido a una sobresaturación de productos de biosíntesis intermedios, como lo son los diacilglicéridos, parece ser un evento clave en la lipotoxicidad inducida por AGS. Por lo tanto, el exceso de ambos ácidos grasos es perjudicial para la función hepática, produciendo hepatotoxicidad, donde el aumento de la formación de radicales libres empeora el daño.

\section{LIPOTOXICIDAD OXIDATIVA}

Se ha sugerido que el aumento de EROs genera lipotoxicidad hepática en la EHGNA, demostrada por el aumento en los niveles séricos de LDL-oxidadas y la peroxidación lipídica sérica (71). La acumulación de AGL estimula la generación de EROs en el hígado, posiblemente debido a la disminución de la $\beta$-oxidación mitocondrial y/o microsomal o por un efecto bloqueador de la cadena de transferencia de electrones mitocondrial (72); afectando la obtención de energía a partir de la oxidación de ácidos grasos. Sin embargo, también se propone que una disminución en el pool de la quinona mitocondrial y en el metabolismo oxidativo mitocondrial, sería la base de la mayor producción de EROs (73). En células hepáticas HepG2 y McNtcp.24 expuestas a AGS se ha reportado despolarización mitocondrial, liberación del citocromo c, aumento en la producción de EROs y la interrupción de la función lisosomal (74); afectando así la digestión intracelular de macromoléculas.

El aumento en la expresión (75) y actividad del citocromo P450 2E1 (CYP2E1; sugerida por desempeñar un papel en la enfermedad hepática alcohólica) (76-77) es otro factor que contribuye al estrés oxidativo y en la lipotoxicidad. EI CYP2E1 es una proteína monooxigenasa integral de membrana del RE y una fuente de compuestos intermediarios oxidantes, incluyendo EROs. La enzima está implicada en la biotransformación de endo/xenobióticos y realiza la hidroxilación de los ácidos grasos n-6 y n-3 (75). La actividad del CYP2E1 se correlaciona positivamente con el IMC y con el grado de esteatosis (78). El CYP2E1 tiene una función importante en la patogénesis del hígado graso, ya que los pacientes con obesidad grave presentan un incremento significativo en la actividad hepática del citocromo (75). Esta enzima está bajo el control de los receptores activados por PPARs que también inducen la $\beta$-oxidación peroxisomal de los ácidos grasos, otra vía prominente en la generación de EROs (79-80). La producción excesiva de EROs favorece la muerte celular a través de la activación de vías de señalización sensibles al estrés, tales como el factor nuclear potenciador de las cadenas ligeras kappa de las células B (NF-k $\beta$ ) el cual media la inducción de citoquinas pro-inflamatorias implicadas en la RI, tales como la interleuquina-1, la interleuquina-6 y el factor de necrosis tumoral-alfa (TNF- $\alpha$ ) (81-82). Por consiguiente, un estrés oxidativo celular generalizado afecta la homeostasis redox del RE (83-84).

\section{LIPOTOXICIDAD Y ESTRÉS}

\section{DEL RETÍCULO ENDOPLASMÁTICO}

El procesamiento de proteínas, una de las funciones del RE (85-86) es afectado por cualquier disfunción grave del organelo (87). Cualquier alteración en la homeostasis del RE que afecte el plegado de las proteínas, promoviendo su depósito en el lumen y dificultando su exportación, conduce al estrés del RE. La acumulación de proteínas inmaduras en el lumen del RE desencadena una compleja red de señalización adaptativa llamada respuesta de proteína desplegada (UPR) que restablece el normal funcionamiento del RE (88). Las proteínas no plegadas reclutan proteínas de unión de inmunoglobulinas (BIP), chaperonas del RE que inician las tres ramas principales de la UPR: i) estimulación de la síntesis de proteínas, ii) expresión de chaperonas y iii) degradación de las proteínas mal plegadas (89). El estrés del RE, proceso caracterizado por un aumento del contenido de proteínas mal plegadas, se favorece la activación de PERK (del inglés "Protein kinase RNA- like endoplasmic reticulum kinase"), proteína que al activarse inicia la fosforilación del factor de 
iniciación eucarionte 2 (eIF2 $\alpha$ ), responsable de reducir la carga proteica atenuendo la traducción y la síntesis del factor de transcripción activado 4 (ATF4), que activa la transcripción de varias proteínas conocidas como blancos de UPR. Cuando la UPR no puede restaurar las funciones por la magnitud del daño generado por el estrés del RE, el destino de la célula es morir por apoptosis (90-91). La apoptosis es estimulada por la inducción de la proteína potenciadora homóloga de unión CCAAT (CHOP) y la activación de quinasa c-Jun $\mathrm{N}$-terminal (JNK). La activación de JNK también es activada por el estrés oxidativo en la lipotoxicidad (92). Además de su actividad pro-apoptótica, interfiere con la señalización de la insulina mediante la fosforilación del sustrato 1 del receptor de insulina (IRS-1) (93), lo que representa un enlace clave entre el estrés del RE y la RI.

El estrés del RE contribuye a la patología de varias enfermedades hepáticas asociadas con la esteatosis (94). La activación de la UPR está implicada en la enfermedad de hígado graso, alcohólico o no alcohólico y en la hepatitis viral. La evidencia experimental y clínica apoya la participación de los ácidos grasos de cadena larga en las disfunciones del RE, especialmente los AGS, donde su exceso origina estrés del RE y lipoapoptosis (95). Se ha identificado el papel de la vía de señalización PERK/ATF4/CHOP en la toxicidad de los AGS, a través de la estimulación del estrés del RE y el desarrollo de lipoapoptosis en líneas celulares de hepatocitos humanos L02 y HepG2 (96). Se ha evidenciado que un nivel alto de palmitato (16:0) exógeno altera la homeostasis de RE mediante la reducción en la expresión de proteína BIP en células HepG2 (97). Por el contrario, la sobreexpresión de la proteína BIP atenúa el estrés en el RE, disminuye la expresión de CHOP y protege las células de la apoptosis inducida por palmitato
(98). Dos de los receptores implicados en el estrés del RE, la quinasa-endonucleasa dependiente de inositol (IRE1 $\alpha$ ) y PERK, se caracterizan por responder al aumento en la saturación de los ácidos grasos (99). Este nuevo mecanismo eplicaría la mayor toxicidad de los AGS versus los ácidos AGI, así como con el efecto protector de UPR frente a la toxicidad por los AGS. Por lo tanto, la alteración del metabolismo lipídico puede provocar estrés del RE y activar la UPR; siendo la alteración de este estado una posible causa de EHGNA.

Es también en esta vía donde los AGPICL n-3 pueden evitar que se inicie la EHGNA, gracias a sus propiedades como estabilizadores y reguladores de la fluidez de la membrana celular (100) (figura 2). Los AGPICL n-3 son un componente esencial que modula la acción de los transportadores y de las enzimas unidas a la membrana. Por lo tanto, su ingesta dietética influye en la composición y función de los fosfolípidos de la membrana de los hepatocitos (101). Además, los bajos niveles de $\mathrm{n}-3$ en los fosfolípidos del músculo esquelético, se asocian a RI (102); uno de los principales factores para el desarrollo de EHGNA.

\section{EFECTOS BENEFICIOSOS DE LOS AGPICL N-3 EN LA EHGNA}

El EPA se ha destacado por su acción protectora de la salud cardiovascular, con efectos hipocolesterolémicos, hipotrigliceridémicos, antinflamatorios, antitrombóticos, antiarrítmicos e hipotensores. El DHA desempeña un rol clave en la protección y conservación del sistema nervioso central, especialmente en el cerebro, en las funciones de neurogénesis, sinaptogénesis, desarrollo del órgano visual, regulación génica, entre otras. Se ha planteado el posible papel nutricional de los AGPICL n-3 frente a la EHGNA. En estudios en ratas con una

\section{AGPICL n-3 (EPA + DHA)}

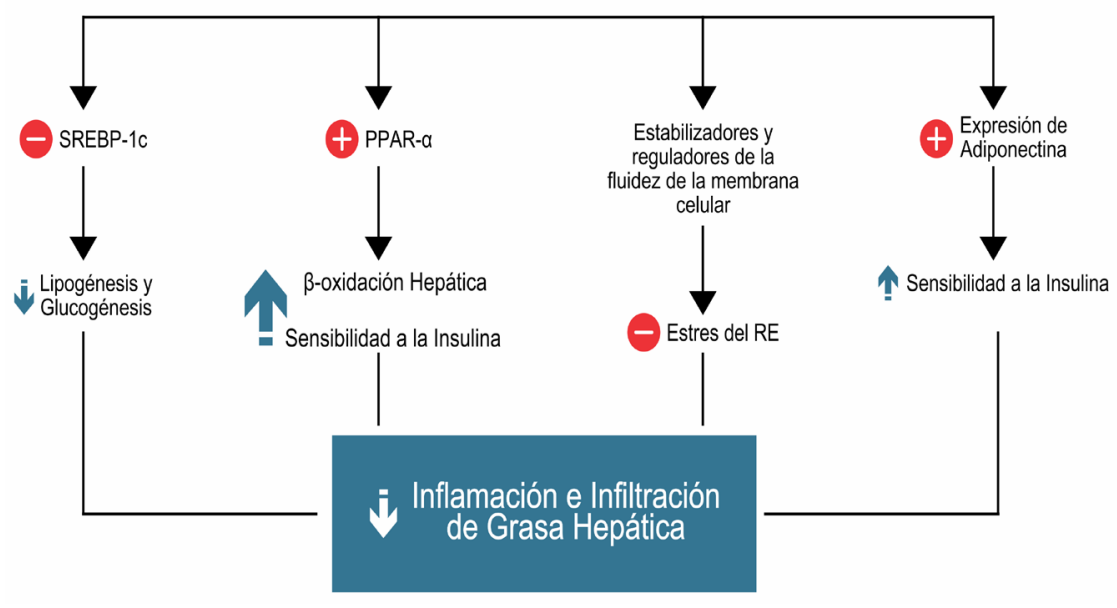


DAG se ha observado una disminución en la biosíntesis y en el contenido de grasa en el hígado. En condiciones de estrés oxidativo hepático inducido por el exceso de alimentación, pacientes obesos con EGHNA presentan dos importantes alteraciones relacionadas con la $\mathrm{RI}$ y el agotamiento hepático de AGPICL $n-3$. Esto se evidencia por una disminución del $50 \%$ en los niveles de EPA y DHA, con aumento de la peroxidación lipídica del hígado y disminución de los antioxidantes en comparación con los controles (103). La reducción hepática de los AGPICL n-3 podría ser causada por varios mecanismos, tales como i) una mayor peroxidación hepática de n-3 debido a la generación de EROs y a su alta susceptibilidad a los ataques de los radicales libres (104) y ii) una desaturación defectuosa de ALA por las enzimas $\Delta-5$ y $\Delta-6$ desaturasa (105), demostrado por la medición directa de la actividad de ambas enzimas que mostró una disminución del $66 \%$ y $87 \%$, respectivamente, en pacientes obesos sobre los controles. El agotamiento de AGPICL n-3 puede favorecer la formación de AGL y síntesis de TAG, alterando la capacidad de oxidación de ácidos grasos, y por consiguiente un exceso de almacenamiento hepático, lo cual ha sido observado en ratas alimentadas con una DAG.

Varios estudios han mostrado el papel fundamental que tienen los AGPICL n-3 en la regulación del metabolismo de los lípidos hepáticos, ejercido a través de la regulación de la expresión y el procesamiento del SREBP-1c que conlleva una menor capacidad lipogénica y glicogénica y que es regulada por PPAR- $\alpha$, que favorece la oxidación de ácidos grasos y reduce los lípidos plasmáticos (figura 2). La sobreexpresión de SREBP-1C induce la EHGNA, a consecuencia de la ingesta de un exceso de calorías procedentes de grasa (106) o de carbohidratos (107), mientras que la administración de AGPICL $n-3$ reduce significativamente la infiltración grasa del hígado (108-109). Los AGPICL n-3 disminuyen la cantidad del SREBP-1c maduro por medio de la proteólisis vía catabolismo proteasómico (110). Por lo tanto, el efecto de los AGPICL n-3 en PPAR- $\alpha$ es aumentar la $\beta$-oxidación hepática y el catabolismo de los lípidos, mientras que su efecto sobre SREBP-1 es reducir la producción de lípidos endógenos (figura 2).

Frente al posible papel de los AGPICL n-3 en la EHGNA, investigadores han puesto de manifiesto y confirmado estos estudios, como es el caso de Capanni et al., (2006) (111) que investigaron los efectos de la suplementación de $1 \mathrm{~g}$ de AGPICL n-3 durante 12 meses en 56 pacientes con EHGNA, donde 42 de ellos se sometieron a suplementación y los 14 restantes fueron grupo control, indicando que los $A G P I C L$ n-3 mejoraron los aspectos bioquímicos de esta enfermedad, disminuyendo las enzimas hepáticas relacionadas directamente con el metabolismo hepático; aspartato transaminasa (AST), alanina aminotransferasa (ALT), Y (gama)-glutamil-transferasa (GGT) y los niveles de TG y glucosa. En el grupo de pacientes suplementados con AGPICL n-3, la ecografía reveló una regresión del daño hepático al final del tratamiento. Los resultados después de la suplementación fueron un $23,8 \%$ de pacientes sin esteatosis, $33,3 \%$ con esteatosis leve, $28,6 \%$ con esteatosis moderada y un $14,3 \%$ con esteatosis grave. Por el contrario, el número de pacientes con hígado graso y el grado de esteatosis se mantuvo sin cambios en el grupo control. Finalmente, el $64 \%$ de los pacientes en el grupo AGPICL n-3 presentó una regresión de la esteatosis hepática a hígado normal. Del mismo modo, una revisión de la literatura realizada por Masterton et al., (2010) (112) encontró que la administración de AGPICL n-3 en animales reduce la esteatosis hepática, mejora la sensibilidad a la insulina y los marcadores bioquímicos de la inflamación, resultados similares a los obtenidos en humanos.

\section{CONCLUSIONES}

La EHGNA es una patología cada vez más común y relevante y de alta prevalencia en la población debido al daño que provoca en el hígado. Los mecanismos son multifactoriales, entre ellos la sobrealimentación con un alto consumo de carbohidratos, AGS y AGT, el SM y la dislipidemia. Estos factores llevan a la RI y a un exceso de AGL, y su almacenamiento en el hígado lo que ocasiona la EHGNA. La sobrecarga lipídica de los AGL generan lipotoxicidad y lipoapoptosis hepática debido al alto consumo de AGS y AGT, responsables de alterar las funciones fisiológicas de las membranas plasmáticas disminuyendo su fluidez. Se genera un aumento de la peroxidación lipídica, estimulando el almacenamiento de TG por la expresión de la actividad de enzimas lipogénicas y glucolíticas. El estrés oxidativo genera lipotoxicidad hepática, debido a la disminución de la $\beta$-oxidación mitocondrial de los ácidos grasos y su acumulación en el hígado. La constante producción de EROs, conduce a la muerte celular a través de la activación de diferentes vías de señalización y citoquinas pro-inflamatorias. El estrés oxidativo afecta la homeostasis del $R E$, produciendo el estrés del organelo, el cual responde con la UPR para restaurar el funcionamiento reticular. En algunas ocasiones al no poder ejecutarse correctamente este proceso, se produce apoptosis activada por la proteína CCAAT y por el receptor JNK. Estos mecanismos son considerados hepatotóxicos y pueden desencadenar EHGNA. Ultimamente los AGPICL $\mathrm{n}-3$ se han considerado como una opción para el tratamiento de la EHGNA, acción consolidada por diversos estudios que demuestran su papel protector en el hígado. Estudios en ratas alimentadas con una DAG suplementadas con AGPICL n-3 demuestran una considerable disminución en la infiltración grasa hepática e inflamación. Otros estudios muestran una regulación del metabolismo lipídico a través de la inhibición del factor de transcripción SREBP-1c que tiene capacidad lipogénica. Igualmente, se ha demostrado que los $A G P I C L$ $\mathrm{n}-3$ pueden mejorar la sensibilidad a la insulina y estimular la $\beta$-oxidación de los ácidos grasos, gracias a su capacidad de actuar como ligando del PPAR- $\alpha$. La suplementación de estos ácidos grasos en diversos animales logró restaurar el ARNm de la adiponectina y mejorar su expresión. Además, con relación al estrés del RE activado por la UPR, que puede ser originado por diferentes causas, entre ellas la reducción de los niveles EPA Y DHA en los fosfolípidos de la membrana celular, los AGPICL n-3 podrían tener un papel protector en este organelo y evitar que se originen alteraciones homeostáticas implicadas en el desarrollo de EHGNA como el estrés RE. Los AGPICL n-3 actúan a través de diferentes mecanismos de acción, siendo el más importante el asociado al metabolismo intracelular, a través de la inhibición de las enzimas lipogénicas, la disminución del almacenamiento de ácidos grasos y el aumento de la ß-oxidación mitocondrial. Además, los AGPICL n-3 aumentan la sensibilidad a la insulina y reduciendo algunos marcadores de inflamación, por lo cual se deben considerar como una alternativa nutricional en la prevención y tratamiento de la EHGNA.

\section{RESUMEN}

La enfermedad por hígado graso no alcohólico (EHGNA) se asocia comúnmente con las características clínicas del síndrome metabólico como la obesidad, resistencia a la insulina y dislipidemia. La importancia clínica se debe a su elevada prevalencia (30\% de la población general) y su amplio espectro de daño histológico que va desde la esteatosis simple generalmente no progresiva, a la esteatohepatitis no alcohólica, que puede conducir a cirrosis, carcinoma hepatocelular, e 
insuficiencia hepática. En la actualidad, se han caracterizado diferentes factores que conllevan a esta enfermedad hepática, destacándose principalmente el alto contenido de ácidos grasos libres y la resistencia a insulina. El exceso de ácidos grasos libres puede desencadenar lipotoxicidad hepática originada por un alto consumo de ácidos grasos saturados, ácidos grasos trans y carbohidratos, así como por un aumento de los radicales libres y del estrés del retículo endoplásmico. En lo que concierne a los ácidos grasos poliinsaturados de cadena larga n-3 (AGPICL n-3), se han atribuido múltiples beneficios para la salud humana. Los AGPICL n-3 EPA y DHA tienen efectos protectores en la salud cardiovascular y en la funcionalidad e integridad del sistema nervioso central. Actualmente el uso nutricional de ambos ácidos grasos es cada vez más amplio, atribuyendo sus efectos positivos no solamente al tratamiento de las enfermedades cardiovasculares y neurodegenerativas, sino también considerándolos una alternativa eficaz en el manejo de nutricional de la EHGNA. El presente trabajo analiza el uso potencial de los AGPICL n-3 en la prevención y manejo nutricional de la EHGNA.

Palabras clave: Enfermedad por hígado graso no alcohólico, dislipidemia, lipotoxicidad, ácidos grasos polinsaturados de cadena larga $n-3$.

Agradecimientos: Los autores agradecen al proyecto de Investigación FONDECYT de Iniciación 11140174 (R. Valenzuela) y al programa Enlaza-Mundos de la Alcaldía de Medellín (Colombia) - Agencia de Educación Superior de MedellínSAPIENCIA por su apoyo a la co-financiación de estudios de postgrado en el extranjero.

Conflictos de interés: Los autores han declarado no tener conflicto de intereses.

\section{BIBLIOGRAFÍA}

1. G. O. Burr and M. M. Burr. A new deficiency disease produced by the rigid exclusion of fat from the diet. J Biol Chem. 1929, 82, 345-67.

2. Hansen $A E$, Haggard ME, Boelsche AN, Adam DJ, Wiese HF. Essential fatty acids in infant nutrition. III. Clinical manifestations of linoleic acid deficiency. J Nutr. 1958; 66(4):565-76.

3. Harris WS. The omega-3 index: from biomarker to risk marker to risk factor. Curr Atheroscler Rep. 2009; 11(6):411-7.

4. Fetterman JW Jr, Zdanowicz MM. Therapeutic potential of $n-3$ polyunsaturated fatty acids in disease, Am J Health Syst Pharm. 2009; 66(13):1169-79.

5. Valenzuela $R$, Tapia $G$ and Valenzuela A. Omega-3 fatty acids (EPA and DHA) and its application in diverse clinical situations. Rev Chil Nutr. 2011 (38):356-67.

6. Hurst S, Zainal Z, Caterson B, Hughes CE, Harwood JL. Dietary fatty acids and arthritis. Prostaglandins Leukot Essent Fatty Acids 2010; 82(4-6);315-8.

7. Oliver E, McGillicuddy F, Phillips C, Toomey S, Roche HM. The role of inflammation and macrophage accumulation in the development of obesity-induced type 2 diabetes mellitus and the possible therapeutic effects of long-chain $n-3$ PUFA. Proc Nutr Soc. 2010; 69(2):232-43.

8. Cederholm T, Palmblad J. Are omega-3 fatty acids options for prevention and treatment of cognitive decline and dementia? Curr Opin Clin Nutr Metab Care 2010; 13(2):150-5.

9. Roth EM, Harris WS. Fish oil for primary and secondary prevention of coronary heart disease. Curr Atheroscler Rep. 2010; 12(1):66-72.

10. Fasano E, Serini S, Piccioni E, Innocenti I, Calviello G. Chemoprevention of lung pathologies by dietary $n-3$ polyunsaturated fatty acids. Curr Med Chem. 2010; 17(29):3358-76.

11. Ferguson $L R$, Smith $B G$, James $B J$. Combining nutrition, food science and engineering in developing solutions to inflammatory bowel diseases--omega-3 polyunsaturated fatty acids as an example. Food Funct. 2010;1(1):60-72.

12. Bassaganya-Riera J, Hontecillas R. Dietary conjugated linoleic acid and n-3 polyunsaturated fatty acids in inflammatory bowel disease. Curr Opin Clin Nutr Metab Care 2010; 13(5):569-73.

13. Szymanski KM, Wheeler DC, Mucci LA. Fish consumption and prostate cancer risk: a review and meta-analysis. Am J Clin Nutr. 2010; 92(5):1223-33.

14. Friedman AN. Omega-3 fatty acid supplementation in advanced kidney disease. Semin Dial 2010; 23(4):396-400.

15. Rodrigo $R$, Cereceda $M$, Castillo $R$, Asenjo $R$, Zamorano $J$, Araya J, et al. Prevention of atrial fibrillation following cardiac surgery: basis for a novel therapeutic strategy based on non-hypoxic myocardial preconditioning. Pharmacol Ther. 2008; 118(1):104-27.

16. Anderson, N. \& Borlak, J. Molecular mechanisms and therapeutic targets in steatosis and steatohepatitis. Pharmacol Rev. 2008; 60: 311-57

17. Cusi K. Role of insulin resistance and lipotoxicity in non-alcoholic steatohepatitis. Clin Liver Dis. 2009; 13(4):545-63.

18. Filozof C, Goldstein BJ, Williams RN, Sanyal A. Non-Alcoholic Steatohepatitis: Limited Available Treatment Options but Promising Drugs in Development and Recent Progress Towards a Regulatory Approval Pathway. Drugs 2015.

19. Byrne CD, Targher G. NAFLD: a multisystem disease. J Hepatol. 2015; 62: S47-64.

20. Ahmed M. Non-alcoholic fatty liver disease in 2015. World J Hepatol. 2015; 7(11):1450-9.

21. Stein DT. Measurement of intracellular triglyceride stores by H spectroscopy: validation in vivo. Am J Physiol. 1999; 276(5 Pt 1):E977-89.

22. Browning JD, Szczepaniak LS, Dobbins $R$, Nuremberg $P$, Horton JD, Cohen JC, et al. Prevalence of hepatic steatosis in an urban population in the United States: impact of ethnicity. Hepatology 2004; 40(6):1387-95.

23. Day CP. Pathogenesis of steatohepatitis. Best Pract Res Clin Gastroenterol. 2002; 16(5):663-78.

24. Gaby AR. Adverse effects of dietary fructose. Altern Med Rev. 2005; 10(4):294-306.

25. Wei $J L$, Leung $J C$, Loong $T C$, Wong GL, Yeung $D K$, Chan RS, Chan HL, Chim AM2, Woo J, Chu WC, Wong VW. Prevalence and Severity of Nonalcoholic Fatty Liver Disease in Non-Obese Patients: A Population Study Using ProtonMagnetic Resonance Spectroscopy. Am J Gastroenterol. 2015; 110(9):1306-14.

26. Cuchel M, Bloedon LT, Szapary PO, Kolansky DM, Wolfe $M L$, Sarkis $A$, et al. Inhibition of microsomal triglyceride transfer protein in familial hypercholesterolemia. $N$ Engl J Med. 2007; 356(2):148-56.

27. Lee RG, Fu W, Graham MJ, Mullick AE, Sipe D, Gattis D, et al. Crooke RM. Comparison of the pharmacological profiles of murine antisense oligonucleotides targeting apolipoprotein $B$ and microsomal triglyceride transfer protein. J Lipid Res. 2013; 54: 602-14.

28. Nissar AU, Tasduq SA. Endoplasmic reticulum stress and 
Oxidative stress in the pathogenesis of Non-alcoholic fatty liver disease. Free Radic Res. 2015;1-32.

29. Zámbó V, Simon-Szabó L, Szelényi P, Kereszturi E, Bánhegyi $G$, Csala M. Lipotoxicity in the liver. World J Hepatol. 2013; 5(10):550-7.

30. Cusi K. Role of insulin resistance and lipotoxicity in non-alcoholic steatohepatitis. Clin Liver Dis. 2009; 13(4):545-63.

31. Tiniakos DG. Nonalcoholic fatty liver disease/nonalcoholic steatohepatitis: histological diagnostic criteria and scoring systems. Eur J Gastroenterol Hepatol. 2010; 22(6): 643-50.

32. Scheen AJ, Luyckx FH. Nonalcoholic steatohepatitis and insulin resistance: interface between gastroenterologists and endocrinologists. Acta Clin Belg. 2003; 58 (2): 81-91.

33. Day CP, James OF. Steatohepatitis: a tale of two "hits"? Gastroenterology 1998; 114(4):842-5.

34. Gastaldelli A, Cusi K, Pettiti M, Hardies J, Miyazaki Y, Berria $R$, et al. Relationship between hepatic/visceral fat and hepatic insulin resistance in nondiabetic and type 2 diabetic subjects. Gastroenterology 2007; 33(2):496-506.

35. Fabbrini E1, Mohammed BS, Magkos F, Korenblat KM, Patterson BW, Klein S. Alterations in adipose tissue and hepatic lipid kinetics in obese men and women with nonalcoholic fatty liver disease. Gastroenterology 2008; 134(2):424-31.

36. Adiels $M$, Taskinen MR, Packard C, Caslake MJ, SoroPaavonen A, Westerbacka J, et al. Overproduction of large VLDL particles is driven by increased liver fat content in man. Diabetologia 2006; 49(4):755-65.

37. Gastaldelli A, Kozakova M, Højlund K, Flyvbjerg A, Favuzzi A, Mitrakou A, et al; RISC Investigators. Fatty liver is associated with insulin resistance, risk of coronary heart disease, and early atherosclerosis in a large European population. Hepatology 2009; 49(5):1537-44.

38. Gastaldelli A. Fatty liver disease: the hepatic manifestation of metabolic syndrome. Hypertens Res. 2010; 33(6):546-7.

39. Tarantino G, Saldalamacchia G, Conca P, Arena A. Nonalcoholic fatty liver disease: further expression of the metabolic syndrome. J Gastroenterol Hepatol. 2007; 22(3):293-303.

40. Finelli $C$, Tarantino $G$. Is visceral fat reduction necessary to favour metabolic changes in the liver? J Gastrointestin Liver Dis. 2012; 21(2):205-8.

41. Donnelly KL, Smith CI, Schwarzenberg SJ, Jessurun J, Boldt MD, Parks EJ. Sources of fatty acids stored in liver and secreted via lipoproteins in patients with nonalcoholic fatty liver disease. J Clin Invest. 2005; 115(5):1343-51.

42. Souza MR, Diniz Mde F, Medeiros-Filho JE, Araújo MS. Metabolic syndrome and risk factors for non-alcoholic fatty liver disease. Arq Gastroenterol. 2012; 49(1):89-96.

43. Alba LM, Lindor K. Review article: Non-alcoholic fatty liver disease. Aliment Pharmacol Ther. 2003; 17(8):977-86.

44. Miles JM, Nelson RH. Contribution of triglyceride-rich lipoproteins to plasma free fatty acids. Horm Metab Res. 2007; 39(10):726-9.

45. Chapman MJ, Ginsberg HN, Amarenco P, Andreotti F, Borén J, Catapano AL, et al; European Atherosclerosis Society Consensus Panel. Triglyceride-rich lipoproteins and highdensity lipoprotein cholesterol in patients at high risk of cardiovascular disease: evidence and guidance for management. Eur Heart J. 2011; 32(11):1345-61.

46. Ortiz-Lopez C, Lomonaco R, Orsak B, Finch J, Chang Z, Kochunov VG, et al. Prevalence of prediabetes and diabetes and metabolic profile of patients with nonalcoholic fatty liver disease (NAFLD). Diabetes Care 2012; 35(4):873-8.

47. Bugianesi E, McCullough AJ, Marchesini G. Insulin re- sistance: a metabolic pathway to chronic liver disease. Hepatology 2005; 42(5):987-100.

48. Morino K, Petersen KF, Shulman GI. Molecular mechanisms of insulin resistance in humans and their potential links with mitochondrial dysfunction. Diabetes 2006; 55:S9-S15.

49. Gastaldelli A, Cusi K, Pettiti M, Hardies J, Miyazaki Y, Berria $R$, et al. Relationship between hepatic/visceral fat and hepatic insulin resistance in nondiabetic and type 2 diabetic subjects. Gastroenterology 2007; 133(2):496-506.

50. Bugianesi E, Gastaldelli A, Vanni E, Gambino R, Cassader $M$, Baldi $S$, et al. Insulin resistance in non-diabetic patients with non-alcoholic fatty liver disease: sites and mechanisms. Diabetologia 2005; 48(4):634-42.

51. Yki-Järvinen $H$. Fat in the liver and insulin resistance. Ann Med 2005; 37(5):347-56.

52. Kotronen A, Yki-Järvinen H. Fatty liver: a novel component of the metabolic syndrome. Arterioscler Thromb Vasc Biol 2008; 28(1):27-38.

53. Marchesini $G$, Bugianesi E, Forlani G, Cerrelli $F$, Lenzi $M$, Manini $R$, et al. Nonalcoholic fatty liver, steatohepatitis, and the metabolic syndrome. Hepatology 2003; 37(4):917-23.

54. Vanni E, Bugianesi E, Kotronen A, De Minicis S, Yki-Järvinen $H$, Svegliati-Baroni $G$. From the metabolic syndrome to NAFLD or vice versa? Dig Liver Dis 2010; 42(5):320-30.

55. Huang $H L$, Lin WY, Lee LT, Wang HH, Lee WJ, Huang KC. Metabolic syndrome is related to nonalcoholic steatohepatitis in severely obese subjects. Obes Surg 2007; 17(11):1457-63.

56. Gastaldelli A, Harrison S, Belfort-Aguiar R, Hardies J, Balas B, Schenker $S$, e al. Pioglitazone in the treatment of NASH: the role of adiponectin. Aliment Pharmacol Ther. 2010; 32(6):769-75.

57. Yamauchi T, Kamon J, Minokoshi Y, Ito Y, Waki H, Uchida $S$, et al. Adiponectin stimulates glucose utilization and fatty-acid oxidation by activating AMP-activated protein kinase. Nat Med. 2002; 8(11):1288-95.

58. Louet JF, Chatelain F, Decaux JF, Park EA, Kohl C, Pineau $T$, et al. Long-chain fatty acids regulate liver carnitine palmitoyltransferase I gene (L-CPT I) expression through a peroxisome-proliferator-activated receptor alpha (PPAR alpha)-independent pathway. Biochem J. 2001; 354(Pt 1):189-97.

59. Reddy JK, Hashimoto T. Peroxisomal beta-oxidation and peroxisome proliferator-activated receptor alpha: an adaptive metabolic system. Annu Rev Nutr. 2001; 21:193-230.

60. Akiyama TE, Nicol CJ, Fievet C, Staels B, Ward JM, Auwerx $J$, et al. Peroxisome proliferator-activated receptor-alpha regulates lipid homeostasis, but is not associated with obesity: studies with congenic mouse lines. Biol Chem. 2001; 276(42):39088-93.

61. Stienstra R, Mandard S, Patsouris D, Maass C, Kersten S, Müller M. Peroxisome proliferator-activated receptor alpha protects against obesity-induced hepatic inflammation. Endocrinology 2007; 148(6):2753-63.

62. Esfahani M, Movahedian A, Baranchi M, Goodarzi MT. Adiponectin: an adipokine with protective features against metabolic syndrome. Iran J Basic Med Sci. 2015; 18(5):430-42.

63. Kawano Y, Cohen DE. Mechanisms of hepatic triglyceride accumulation in non-alcoholic fatty liver disease. J Gastroenterol. 2013; 48(4):434-41.

64. Ochiai M, Fujii K, Takeuchi H, Matsuo T. Effects of dietary trans fatty acids on fat accumulation and metabolic rate 
in rat. J Oleo Sci. 2013; 62(2):57-64.

65. Obara N, Fukushima $K$, Ueno Y, Wakui Y, Kimura O, Tamai $K$, et al. Possible involvement and the mechanisms of excess trans-fatty acid consumption in severe NAFLD in mice. $J$ Hepatol. 2010; 53(2):326-34.

66. Machado RM, Stefano JT, Oliveira CP, Mello ES, Ferreira $F D$, Nunes VS, et al. Intake of trans fatty acids causes nonalcoholic steatohepatitis and reduces adipose tissue fat content. J Nutr. 2010; 140(6):1127-32.

67. Dhibi M, Brahmi F, Mnari A, Houas Z, Chargui I, Bchir L, et al. The intake of high fat diet with different trans fatty acid levels differentially induces oxidative stress and non alcoholic fatty liver disease (NAFLD) in rats. Nutr Metab (Lond) 2011; 8(1):65.

68. Shimano H. SREBPs: physiology and pathophysiology of the SREBP family. FEBS J. 2009; 276(3):616-21.

69. Mantzaris MD, Tsianos EV, Galaris D. Interruption of triacylglycerol synthesis in the endoplasmic reticulum is the initiating event for saturated fatty acid-induced lipotoxicity in liver cells. FEBS J. 2011; 278(3):519-30.

70. Zhang Y, Dong L, Yang X, Shi H, Zhang L. $\alpha$-Linolenic acid prevents endoplasmic reticulum stress-mediated apoptosis of stearic acid lipotoxicity on primary rat hepatocytes. Lipids Health Dis. 2011; 10:81.

71. Chalasani N, Deeg MA, Crabb DW. Systemic levels of lipid peroxidation and its metabolic and dietary correlates in patients with nonalcoholic steatohepatitis. Am J Gastroenterol. 2004; 99(8):1497-502.

72. Seifert EL, Estey C, Xuan JY, Harper ME. Electron transport chain-dependent and -independent mechanisms of mitochondrial $\mathrm{H} 2 \mathrm{O} 2$ emission during long-chain fatty acid oxidation. J Biol Chem. 2010; 285(8):5748-58.

73. Vial G, Dubouchaud H, Couturier K, Cottet-Rousselle C, Taleux N, Athias A, et al. Effects of a high-fat diet on energy metabolism and ROS production in rat liver. $J$ Hepatol. 2011; 54(2):348-56

74. Li Z, Berk M, Mclntyre TM, Gores GJ, Feldstein AE. The lysosomal-mitochondrial axis in free fatty acid-induced hepatic lipotoxicity. Hepatology 2008; 47(5):1495-503.

75. Weltman MD, Farrell GC, Hall $P$, Ingelman-Sundberg $M$, Liddle C. Hepatic cytochrome P450 2E1 is increased in patients with nonalcoholic steatohepatitis. Hepatology 1998; 27(1):128-33.

76. Videla $L A$, Rodrigo $R$, Orellana $M$, Fernandez $V$, Tapia $G$, Quiñones $L$, et al. Oxidative stress-related parameters in the liver of non-alcoholic fatty liver disease patients. Clin Sci (Lond) 2004; 106(3):261-8.

77. Orellana $M$, Rodrigo $R$, Varela $N$, Araya J, Poniachik J, Csendes $A$, et al. Relationship between in vivo chlorzoxazone hydroxylation, hepatic cytochrome P450 2 E1 content and liver injury in obese non-alcoholic fatty liver disease patients. Hepatol Res. 2006; 34(1):57-63.

78. Chtioui H, Semela D, Ledermann M, Zimmermann A, Dufour JF. Expression and activity of the cytochrome P450 2E1 in patients with nonalcoholic steatosis and steatohepatitis. Liver Int. 2007; 27(6):764-71.

79. Hardwick JP, Osei-Hyiaman D, Wiland H, Abdelmegeed $M A$, Song BJ. PPAR/RXR Regulation of Fatty Acid Metabolism and Fatty Acid omega-Hydroxylase (CYP4) Isozymes: Implications for Prevention of Lipotoxicity in Fatty Liver Disease. PPAR Res. 2009; 2009:952734.

80. Gao D, Nong S, Huang X, Lu Y, Zhao H, Lin Y, et al. The effects of palmitate on hepatic insulin resistance are mediated by NADPH Oxidase 3-derived reactive oxygen species through JNK and p38MAPK pathways. J Biol Chem. 2010; 285(39):29965-73.

81. Klaunig JE, Kamendulis LM, Hocevar BA. Oxidative stress and oxidative damage in carcinogenesis. Toxicol Pathol. 2010; 38(1):96-109.

82. Ghosh J, Das J, Manna P, Sil PC. Taurine prevents arsenicinduced cardiac oxidative stress and apoptotic damage: role of NF-kappa B, p38 and JNK MAPK pathway. Toxicol Appl Pharmacol. 2009; 240(1):73-87.

83. Bánhegyi G, Margittai E, Szarka A, Mandl J, Csala M. Crosstalk and barriers between the electron carriers of the endoplasmic reticulum. Antioxid Redox Signal 2012; 16(8):772-80.

84. Piccirella S, Czegle I, Lizák B, Margittai E, Senesi S, Papp E, et al. Uncoupled redox systems in the lumen of the endoplasmic reticulum. Pyridine nucleotides stay reduced in an oxidative environment. J Biol Chem. 2006; 281(8):4671-7.

85. Csala M, Kereszturi É, Mandl J, Bánhegyi G. The endoplasmic reticulum as the extracellular space inside the cell: role in protein folding and glycosylation. Antioxid Redox Signal 2012; 16(10):1100-8.

86. Csala M, Bánhegyi G, Benedetti A. Endoplasmic reticulum: a metabolic compartment. FEBS Lett 2006; 580(9):2160-5.

87. Mandl J, Mészáros T, Bánhegyi G, Csala M. Minireview: endoplasmic reticulum stress: control in protein, lipid, and signal homeostasis. Mol Endocrinol. 2013; 27(3):384-93.

88. Nissar AU, Tasduq SA. Endoplasmic reticulum stress and Oxidative stress in the pathogenesis of Non-alcoholic fatty liver disease. Free Radic Res. 2015; 49(12):1405-18.

89. Mandl J, Mészáros T, Bánhegyi G, Hunyady L, Csala M. Endoplasmic reticulum: nutrient sensor in physiology and pathology. Trends Endocrinol Metab. 2009; 20(4):194-201.

90. Lei X, Zhang S, Bohrer A, Ramanadham S. Calcium-independent phospholipase A2 (iPLA2 beta)-mediated ceramide generation plays a key role in the cross-talk between the endoplasmic reticulum (ER) and mitochondria during $E R$ stress-induced insulin-secreting cell apoptosis. J Biol Chem. 2008; 283(50):34819-32.

91. Joyce $M A$, Walters $K A$, Lamb SE, Yeh MM, Zhu LF, Kneteman $N$, et al. HCV induces oxidative and ER stress, and sensitizes infected cells to apoptosis in SCID/Alb-uPA mice. PLoS Pathog. 2009;5(2):e1000291.

92. Gao D, Nong S, Huang $X, L u Y$, Zhao $H$, Lin $Y$, et al. The effects of palmitate on hepatic insulin resistance are mediated by NADPH Oxidase 3-derived reactive oxygen species through JNK and p38MAPK pathways. J Biol Chem. 2010; 285(39):29965-73.

93. Aguirre $V$, Uchida $T$, Yenush $L$, Davis $R$, White MF. The c-Jun $\mathrm{NH}(2)$-terminal kinase promotes insulin resistance during association with insulin receptor substrate-1 and phosphorylation of Ser(307). J Biol Chem. 2000 Mar; 275(12):9047-54.

94. Zhang $X Q, X u C F, Y u C H$, Chen $W X, L i$ YM. Role of endoplasmic reticulum stress in the pathogenesis of nonalcoholic fatty liver disease.World J Gastroenterol. 2014; 20(7):1768-76.

95. Cao J, Dai DL, Yao L, Yu HH, Ning B, Zhang Q, et al. Saturated fatty acid induction of endoplasmic reticulum stress and apoptosis in human liver cells via the PERK/ ATF4/CHOP signaling pathway. Mol Cell Biochem. 2012; 364(1-2):115-29.

96. Gu X, Li K, Laybutt DR, He ML, Zhao HL, Chan JC, Xu $G$. Bip overexpression, but not CHOP inhibition, attenuates fatty-acid-induced endoplasmic reticulum stress and 
apoptosis in HepG2 liver cells. Life Sci. 2010; 87(2326):724-32.

97. Ariyama $H$, Kono N, Matsuda S, Inoue T, Arai H. Decrease in membrane phospholipid unsaturation induces unfolded protein response. J Biol Chem. 2010; 285(29):22027-35.

98. Kitai Y, Ariyama H, Kono N, Oikawa D, Iwawaki T, Arai $H$. Membrane lipid saturation activates IRE1 $\alpha$ without inducing clustering. Genes Cells 2013; 18(9):798-809.

99. Volmer $R$, van der Ploeg K, Ron D. Membrane lipid saturation activates endoplasmic reticulum unfolded protein response transducers through their transmembrane domains. Proc Natl Acad Sci U S A 2013; 110(12):4628-33.

100. Valentine RC, Valentine DL. Omega-3 fatty acids in cellular membranes: a unified concept. Prog Lipid Res. 2004; 43(5):383-402.

101. Clamp AG, Ladha S, Clark DC, Grimble RF, Lund EK. The influence of dietary lipids on the composition and membrane fluidity of rat hepatocyte plasma membrane. Lipids 1997; 32(2):179-84.

102. Borkman M, Storlien $L H$, Pan DA, Jenkins AB, Chisholm $D J$, Campbell LV. The relation between insulin sensitivity and the fatty-acid composition of skeletal-muscle phospholipids. N Engl J Med. 1993; 328(4):238-44.

103. Allard JP, Aghdassi E, Mohammed S, Raman M, Avand $G$, Arendt BM, et al. Nutritional assessment and hepatic fatty acid composition in non-alcoholic fatty liver disease (NAFLD): a cross-sectional study. J Hepatol. 2008; 48(2):300-7.

104. Sevanian A, Hochstein P. Mechanisms and consequences of lipid peroxidation in biological systems. Annu Rev Nutr. 1985; 5:365-90.

105. Araya J, Rodrigo R, Videla $L A$, Thielemann $L$, Orellana $M$, Pettinelli $P$, et al. Increase in long-chain polyunsaturated fatty acid $n-6 / n-3$ ratio in relation to hepatic steatosis in patients with non-alcoholic fatty liver disease. Clin Sci
(Lond) 2004; 106(6):635-43.

106. Biddinger SB, Almind K, Miyazaki M, Kokkotou E, Ntambi JM, Kahn CR. Effects of diet and genetic background on sterol regulatory element-binding protein-1C, stearoylCoA desaturase 1, and the development of the metabolic syndrome. Diabetes 2005; 54(5):1314-23.

107. Nagai Y, Yonemitsu S, Erion DM, Iwasaki T, Stark $R$, Weismann $D$, et al. The role of peroxisome proliferator-activated receptor gamma coactivator-1 beta in the pathogenesis of fructose-induced insulin resistance. Cell Metab. 2009; 9(3):252-64.

108. Liang G, Yang J, Horton JD, Hammer RE, Goldstein JL, Brown MS. Diminished hepatic response to fasting/refeeding and liver $X$ receptor agonists in mice with selective deficiency of sterol regulatory element-binding protein-1C. J Biol Chem. 2002; 277(11):9520-8.

109. Teran-Garcia M, Adamson AW, Yu G, Rufo C, Suchankova $G$, Dreesen TD, et al. Polyunsaturated fatty acid suppression of fatty acid synthase (FASN): evidence for dietary modulation of NF-Y binding to the Fasn promoter by SREBP-1C. Biochem J. 2007; 402(3):591-600.

110. Lee JN, Zhang X, Feramisco JD, Gong Y, Ye J. Unsaturated fatty acids inhibit proteasomal degradation of Insig-1 at a postubiquitination step. I Biol Chem. 2008; 283(48):33772-83.

111. Capanni M, Calella F, Biagini $M R$, Genise $S$, Raimondi $L$, Bedogni G, Svegliati-Baroni G, Sofi F, Milani S, Abbate $R$, Surrenti $C$, Casini A. Prolonged n-3 polyunsaturated fatty acid supplementation ameliorates hepatic steatosis in patients with non-alcoholic fatty liver disease: a pilot study. Aliment Pharmacol Ther 2006; 23(8):1143-51.

112. Masterton GS, Plevris JN, Hayes PC. Review article: omega-3 fatty acids - a promising novel therapy for nonalcoholic fatty liver disease. Aliment Pharmacol Ther 2010; 31(7):679-92. 